\title{
An Expedient, Generalizable and Realistic Data-Driven Indoor Propagation Model
}

This paper was downloaded from TechRxiv (https://www.techrxiv.org).

\section{LICENSE}

CC BY 4.0

SUBMISSION DATE / POSTED DATE

$21-12-2021 / 22-12-2021$

\section{CITATION}

Bakirtzis, Stefanos Sotirios; Chen, Jiming; Qiu, Kehai; Zhang, Jie; Wassell, lan (2021): An Expedient, Generalizable and Realistic Data-Driven Indoor Propagation Model. TechRxiv. Preprint. https://doi.org/10.36227/techrxiv.17306384.v1

$\mathrm{DOI}$

10.36227/techrxiv.17306384.v1 


\title{
An Expedient, Generalizable and Realistic Data-Driven Indoor Propagation Model
}

\author{
Stefanos Bakirtzis, Member, IEEE, Jiming Chen, Kehai Qiu, Student Member, IEEE, Jie Zhang, Senior \\ Member, IEEE, and Ian Wassell
}

\begin{abstract}
Efficient and realistic indoor radio propagation modelling tools are inextricably intertwined with the design and operation of next generation wireless networks. Machine learning (ML)-based radio propagation models can be trained with simulated or real-world data to provide accurate estimates of the wireless channel characteristics in a computationally efficient way. However, most of the existing research on ML-based propagation models focuses on outdoor propagation modelling, while indoor data-driven propagation models remain site-specific with limited scalability. In this paper we present an efficient and credible ML-based radio propagation modelling framework for indoor environments. Specifically, we demonstrate how a convolutional encoder-decoder can be trained to replicate the results of a ray-tracer, by encoding physics-based information of an indoor environment, such as the permittivity of the walls, and decode it as the path-loss (PL) heatmap for an environment of interest. Our model is trained over multiple indoor geometries and frequency bands, and it can eventually predict the PL for unknown indoor geometries and frequency bands within a few milliseconds. Additionally, we illustrate how the concept of transfer learning can be leveraged to calibrate our model by adjusting its pre-estimate weights, allowing it to make predictions that are consistent with measurement data.
\end{abstract}

Index Terms-Machine Learning, deep learning, indoor radio communication, radio propagation, ray tracing, $\mathbf{5 G}$.

\section{INTRODUCTION}

D URING the past decades, wireless communication systems have experienced immense growth and they are now an indispensable part of our every day life. With the advent of the Internet of Things (IoT), and the establishment of $5 \mathrm{G}$ and beyond systems, the landscape in the wireless ecosystem is expected to change radically [1]-[3]. Unlike legacy communication systems, next generation wireless networks are anticipated to host a vast number of technologies and applications with diverse requirements. Hence, efficient network planning and optimization is becoming a much more convoluted and manifold problem, rendering conventional communication network design techniques obsolete [4].

This work was supported by European Commission through the Horizon 2020 Framework Programme, H2020-MSCA-ITN-2019, MSCA-ITN-EID, Proposal No. 860239, BANYAN.

Stefanos Bakirtzis, Kehai Qiu and Dr. Ian Wassell are with the Department of Computer Science and Technology, University of Cambridge, Cambridge, CB3 0FD, United Kingdom (e-mail: ssb45@cam.ac.uk; kq218@cam.ac.uk ijw24@cam.ac.uk;).

Professor Jie Zhang is with the Department of Electronic and Electrical Engineering, University of Sheffield, Sheffield, S10 2TN, United Kingdom (e-mail: jie.zhang@sheffield.ac.uk).

Dr. Jiming Chen is with RANPLAN Wireless Network Design, 25 Park Street West, Luton, Bedfordshire, LU1 3BE, United Kingdom (e-mail: jiming.chen@ ranplanwireless.com).
Artificial intelligence is a key-enabler towards the reshaping of wireless networks and the shifting from a reactive to a proactive network design approach [5]. In particular, the potential of machine learning (ML) algorithms, a subfield of artificial intelligence, to process large volumes of data and extract purposeful information, can revolutionize wireless network operation [6]. Until now, ML has been employed to tackle a wide range of wireless network design-related problems, such as resource allocation, user mobility analysis, localization, and wireless channel modelling [7]-[9]. The latter case has recently attracted significant interest, as radio propagation modelling is the cornerstone of the cellular network design [10], [11].

Conventionally, for radio channel modeling, empirical or deterministic models are used. Empirical channel models, such as the COST-231 model [12], are derived by fitting measurement campaign data to simplistic mathematical models [13], [14]. Although these models are practical in use, they can demonstrate significant deviations from the actual received path loss values [15], potentially rendering them unreliable. Deterministic models rely on the governing laws of electromagnetic wave propagation, providing an approximate solution to Maxwell's equations. Commonly used deterministic models include ray tracing, the Finite-Difference Time-Domain method or the vector parabolic equation method [16]. Unlike empirical models, deterministic models are site-specific, solving Maxwell's equations within a specified physical geometry, thereby yielding more accurate results.

For that reason, the popularity of deterministic models has grown constantly over recent years. Among the various deterministic models, ray tracing has been widely employed to calculate radio channels characteristics, and it is expected to have a leading role in the deployment and the design of $5 \mathrm{G}$ and beyond systems [17]. A key difference between 5G and legacy communication system design is that a significant part of 5G radio access networks will be installed in indoor environments. Traditionally, in-building traffic is served by outdoor cells, following an outside-in approach, and until now only a small number of buildings have dedicated indoor mobile networks. However, in the $5 \mathrm{G}$ and beyond era, the majority of in-building mobile traffic will be served by indoor base stations or access points [18]. Thus, an accurate and expedient indoor propagation modeling tool is now more important than ever.

Computational efficiency usually appears as a bottleneck for ray tracing, due to the substantial simulation time and memory required to trace all the ray paths, when the number of scattering objects and ray intersections within the simulated space 
increases. Data-driven approaches aim to alleviate this limitation by integrating ray tracing simulators with ML algorithms which are capable of learning and inferring radio propagation parameters [10], [11]. In particular, artificial neural networks (ANNs) have been widely used in an effort to expedite [19] or even replace ray tracing simulators [20]. The preponderance of past research concentrates on urban propagation scenarios [20]-[23], however as has been mentioned, indoor propagation modeling is of high significance in the deployment of 5G networks. Currently, most of the existing approaches on indoor propagation modeling are confined to the use of simple multilayer perceptrons (MLPs) [24] to determine the radio channel characteristics [19], [25]. That poses limitations in terms of the model's generalizability, as MLPs trained within a certain geometry are agnostic to the characteristics of other geometries (materials, building layout, transmitter position) [26]. Additionally, it is necessary to increase the fidelity of ML-based propagation models by considering real measured data, instead of using simulated data only [23], [26], [27].

The main contribution of this paper is to tackle these two fundamental limitations of indoor ML-based propagation models. To that end, first we outline how a convolutional encoderdecoder can be trained to predict the path-loss for an arbitrarily complex indoor environment over multiple frequency bands. In particular, we employ a modified version of the U-Net architecture using stacked dilated convolutions (SDU-Net) [28], which learns to transform an input tensor, comprising information regarding the physical properties of an indoor environment, to a path loss (PL) heatmap. Unlike [19], [26], [27], the predictions of the proposed data-driven framework are based on the electromagnetic properties of the physical environment, such as the permitivity and the conductivity of the walls, and other parameters that affect wave propagation, e.g., the distance and the frequency. We demonstrate that our approach overcomes the problem of limited generalizability, as it is directly applicable to multiple indoor geometries and frequencies, without any further training. The predictions made by our model can replicate closely the results of a ray tracing simulator, with the additional advantage of a substantially reduced computational time (less than a second in a GPU).

To tackle the second limitation, in this paper we present an approach that allows making predictions that match realistic measured data. Thence, we employ the idea of transfer learning to calibrate the proposed data-driven propagation model. Specifically, we introduce an expedient method that allows our model to adjust its pre-estimated weights, computed using simulated data, and provide realistic estimates of the signal level using only a very small quantity of measured data. To summarize, in this paper we show how to leverage the results from a ray tracing simulator to create a generalizable data-driven propagation model for indoor environments. Once trained, our model can be used as a standalone propagation solver to predict the PL for unknown indoor geometries and frequencies. Consequently, it can be readily calibrated to provide realistic estimates of the signal level for any indoor environment, using only a few measurements.

The outline of this paper is as follows. First, in Section II, we discuss the functionality of some basic ANN architectures. In section III, we provide a brief overview of some of the existing approaches to ML-based propagation modeling, focusing on ray tracing. In section IV, we present the proposed data-driven indoor propagation modelling framework. Next, in Section V, we provide numerical results comparing our model with synthetic data computed through a ray tracing simulator. We study scenarios where the proposed framework is trained over multiple geometries and frequency bands, and we also explore its applicability to unknown indoor environments and frequencies. Then, in Section VI, we outline how transfer learning can be exploited to calibrate our model, and we compare the predictions of our and other propagation models to measured data. Finally, Section VII concludes the paper outlining its main contributions.

\section{THEORETICAL BACKGROUND}

Artificial neural networks seek to imitate human intelligence by allowing simple learning components to perform basic computational operations and connect to other learning components. The learning components are referred to as nodes, artificial neurons or just neurons. In what follows we briefly discuss the functionality of MLPs and convolutional neural networks (CNNs), focusing on the latter since in this work we are employing a convolutional encode-decoder.

\section{A. Multilayer perceptrons}

In MLPs, the neurons are arranged into layers, where each neuron has input connections originating from the previous layer and output connections pointing towards the next layer. A typical MLP consists of an input layer, a number of hidden layers, and an output layer, as show in Fig 1, where $x_{1}, x_{2}, \ldots, x_{n}$ and $y_{1}, y_{2}, \ldots, y_{m}$ are the input features and the outputs of the MLP, respectively. With the term input features we refer to the characteristic physical quantities that affect a quantity of interest (QoI), which is the output of the MLP. For instance, commonly used input features of ML propagation models are the operating frequency, the distance, or the transmitter height, while typical QoIs are the signal level or the PL. The hidden layers consist of neurons that apply non-linear transformations to their input data. Specifically, the output, $u_{j}^{(l)}$, of the $j$-th neuron in the $l$-th layer is computed by applying a non-linear function, $g$, to the weighted sum of the previous layer neuron outputs, plus a bias term, $b_{j}^{(l)}$ :

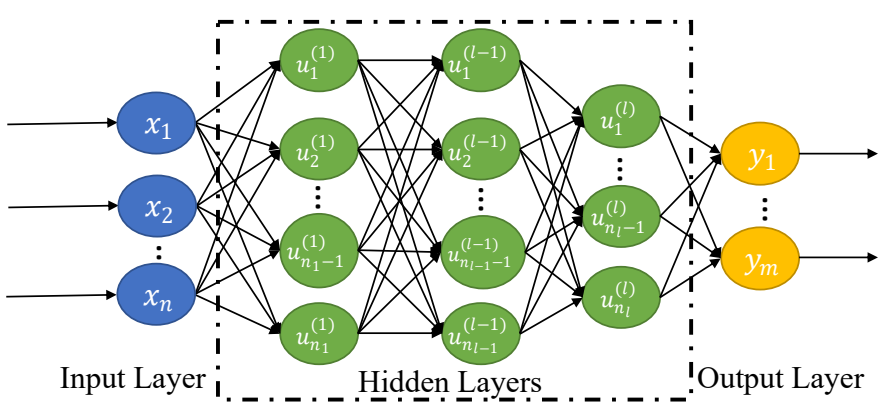

Fig. 1: Multilayer perceptron. 


$$
u_{j}^{(l)}=g\left(\sum_{i}^{n_{l-1}} w_{i, j} u_{i}^{(l-1)}+b_{j}^{(l)}\right)
$$

where $u_{i}^{(l-1)}$ is the output of the $i$-th neuron of the previous layer, $w_{i, j}$ is the weight associated with $u_{i}^{(l-1)}$, and $n_{l-1}$ is the number of neurons in the previous layer. Some commonly used non-linear functions, $g$, are the rectified linear unit (ReLU), the tanh, and the sigmoid function [24]. The output of the MLP is estimated in a similar manner and the QoI can assume discrete categorical or continuous values, for classification and regression problems, respectively.

\section{B. Convolutional neural networks}

Convolutional neural networks have been widely used in computer vision and in image processing-related applications. In CNNs the input data are represented as tensors, and they have three dimensions: the width, $W$, the height, $H$, and the number of channels, $C$. A CNN typically comprises three different layer types: convolutional, pooling, and fully connected layers. An example of a CNN is shown in Fig. 2, where an RGB image, representing the geometry of an indoor environment, is fed to a convolutional layer.

In a convolutional layer, the input tensor is convolved with $n_{f}$ different filters. Each filter identifies different hidden features and it consists of stacked two-dimensional (2D) kernels, i.e., 2D square $f_{w} \times f_{h}$ weight matrices. The number of the stacked kernels is equal to the number of the input channels $C$, and the number of the output channels is equal to the number, $n_{f}$, of the individual filters convolved with the input tensor. The result of these convolutions, i.e., the layer output, is known as the feature map. To estimate the elements of the feature map, each kernel is slid with a stride, $s$, over the respective channel of the input tensor, and the dot product between the filter kernel weights and the points of the corresponding overlapping channel sub-area is computed. Consequently, the elements of the output feature map are computed as the crosschannel summation of the results of the aforementioned dot products. With respect to Fig. 2, that corresponds to computing the dot product between the orange weight boxes and the pixel intensity values of the overlapping sub-area, and then adding the dot products estimated for each one of the 3 RGB channel. Let $X$ be the input tensor and $w$ the weights of the $o$-th filter, then the elements $z$ of the feature map are computed via [24]:

$$
z_{m, n, o}=\sum_{k} \sum_{i} \sum_{j} w_{i, j, k} \cdot X_{m \times s-i, n \times s-j, k}
$$

where the summation over $i$ and $j$ captures spatial correlations between the elements of the same channel, while the summation over $k$ enables unveiling correlations between the different channels of the input tensor (cross-channel correlations). The convolutional layers of a $\mathrm{CNN}$ are typically followed by pooling layers used to simplify the representation of the encoded feature maps through downsampling, and to reduce the number of the model's trainable parameters. The most common pooling operation is max-pooling. In a max-pooling layer, a single $f_{w} \times f_{h} \times f_{c}$ filter, where $f_{c}$ is the number of the input tensor channels, is slid over the received input tensor, and the elements of the output feature map are estimated as the maximum element of the overlapping sub-area of each channel. For instance, the different coloured squares in Fig. 2 correspond to the kernels of the filter used to extract the maximum point from a sub-area of each channel. Finally, CNNs are commonly terminated with fully connected layers, i.e., MLPs, that are used to estimate the network output.

The main advantages of CNNs are the weight sharing and the connection sparsity properties. The first means that the same filter weights are applied to different parts of the input tensor, i.e., the same orange box is slid over the entire indoor geometry in Fig. 2. Thus, each convolutional filter can identify certain kinds of hidden features, and more importantly it is not required to compute different weights for every point of the input tensor. The second property signifies that unlike MLPs, where each output neuron receives information from all the neurons of the previous layer, in CNNs, each neuron considers information originating only from a small sub-area of the previous layer. Thus, the numerical operations required to compute the output value of a neuron decrease substantially. For instance, for the estimation of $z_{1,1,1}$ in Fig. 2 (purple box), only the top-left side of the indoor geometry is considered.

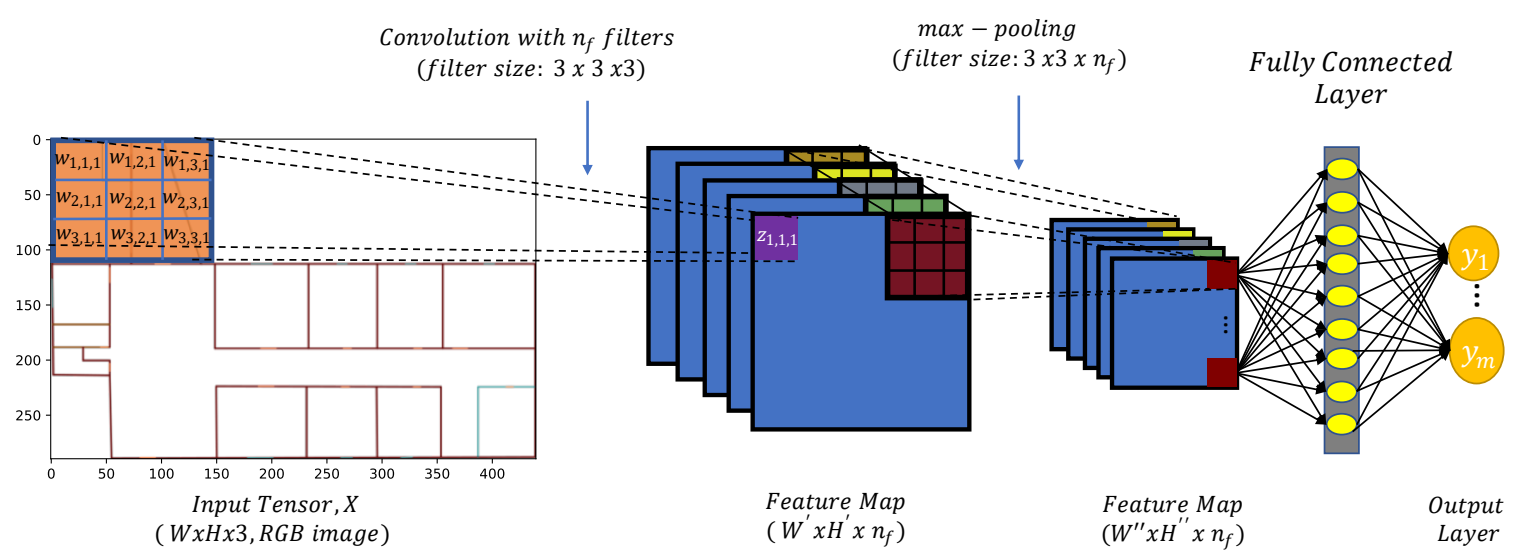

Fig. 2: Typical operations in a CNN. The orange box depicts the kernel of a convolutional filter slid over an RGB image. The different coloured squares represent the kernels of a max-pooling filter used to extract the maximum point from a sub-area of each channel. 
The connection sparsity property also gives rise to the concept of the receptive field, which depicts the information region of the input tensor that affects the output response of a neuron. Evidently, since each convolutional layer encodes and compresses information of the previous layer, the neurons of deeper layers have an indirect access to a larger area of the input tensor, and thereby a larger receptive field [24]. Hence, the shallower layers within a CNN can detect low-level features of the input tensor, whereas the deeper layers, due to their augmented receptive field, can identify more complicated abstract high-level features.

\section{RELATED WORK}

The idea of employing ANNs to improve the performance of radio propagation modeling tools is not new. In [21], the authors used an MLP to calibrate a ray-tracer with measurement taken from three different cities taken at $900 \mathrm{MHz}$ and $1800 \mathrm{MHz}$. The MLP received as input the received signal strength (RSS) provided by the ray-tracer and several simulation parameters (transmitter height, number of reflections and the land type). Then the authors tried to fit the simulated RSS to the measured RSS values and calibrate the ray-tracer. More recently, due to their computational efficiency, the use of CNNs for radio propagation modeling has become popular [20], [23]. In [23], a CNN was trained with images that depicted the buildings of a city, assuming different pixel intensity values according to the building height. Consequently, the PL for different urban environments was simulated through a ray tracing simulator. The city images along with the respective simulated PL values were used as the input and the expected output of the data-driven model, respectively. A more advanced and complete approach was presented in [20], using a U-Net convolutional encoder-decoder. The authors incorporated multiple features to their model by encoding additional information, using seven input channels. Specifically, they included information regarding the surface type, the altitude of each point, the horizontal and the vertical angle difference between each pixel and the antenna position, the antenna height and the operating frequency. Although these models provided accurate results and they overcame the scalability limitation posed by MLPs, they focus on outdoor propagation modeling and they are not applicable to indoor environments.

Most of the existing AI-based indoor propagation models make use of MLPs [19], [25]. The motivation in the work done in [19] was to reduce the computational cost of ray tracing through a coarse-to-dense grid MLP-assisted scheme. A ray tracing simulation was conducted in an indoor environment assuming a coarse grid discretization. Then, the ray tracing results were used to train an MLP to recognize radio channel characteristics and infer the RSS for other points and coverage "holes", assuming a dense grid discretization. In [25], a similar approach was implemented using real measurements instead of ray tracing data. A CNN-based formulation was presented in [27], consisting of two convolutional layers and four fully connected layers, aiming to evaluate the characteristics of a millimeter-wave channel. The input data comprised the coordinates of the receiver and the transmitter, while the output vector included various channel characteristics (PL, delay spread, angle of arrival, etc.). The main drawback all the previous approaches share is that they attempt to associate the geometrical coordinates of an indoor environment to telecommunication-related features. As it was shown in [26], that constitutes a fundamental bottleneck in the applicability of such models in geometries other those in which they are trained.

\section{Problem Formulation And Proposed Model}

In this section we present the details of our data-driven indoor propagation model, called EM DeepRay. We describe the format of the input and output data, the general framework pipeline, and the convolutional encoder-decoder used.

\section{A. Input features and target output}

Wave propagation is affected by four basic mechanisms: reflection, transmission, diffraction and diffuse scattering [16], [29]. It is well-known that the impact of these mechanisms depends on the wavelength $\lambda$ of the propagating wave. In particular, reflections and transmissions require that the objects found within the propagation environment are electrically large, i.e., their dimensions are much larger than $\lambda$. When $\lambda$ is much larger than the object (sharp edges, small openings), the propagating waves will bend around the object and diffraction will occur. Finally, diffuse scattering requires that abrupt variations of the surface height are an order larger than the wavelength. The impact of these mechanisms also depends on the material proprieties, which in turn are related to the radio signal frequency [30].

The electromagnetic properties of a material can be quantified by its permittivity and its permeability. In this work, we assume that all the construction materials found within the simulated indoor geometries are non-magnetic, i.e., they have a constant permeability equal to $\mu_{0}$. The permittivity is formulated as: $\epsilon=\epsilon_{r} \epsilon_{0}=\left(\epsilon_{r}^{\prime}-j \frac{\sigma}{\epsilon_{0} \omega}\right) \epsilon_{0}$, where $\epsilon_{r}$ and $\sigma$ are the relative permittivity and the conductivity, repsectively. Thus, to consider how different materials affect wave propagation we use two input channels that convey information regarding the relative permittivity. Specifically, the first channel depicts the real part of $\epsilon_{r}$ at every point of the simulated grid, and the second includes the value of the conductivity. An example of these channels is shown in Fig. 3a and Fig. 3b, respectively. The conductivity is modeled as $\sigma=c f^{d}$, where $f$ is the frequency of the propagating wave in $\mathrm{GHz}$. The values of $\epsilon_{r}^{\prime}, c$ and $d$, are derived from the ITU-R P.2040-1 Recommendation [30], and they are shown in Table. I. The first channel allows our model to understand the presence of an object and infer the strength of the reflected and the transmitted components of an electromagnetic wave impinging onto it. The second channel accounts for the attenuation that an electromagnetic wave undergoes while it propagates through an absorbing medium. Also, to indicate the transmitter position within the simulated grid, the values of $\epsilon_{r}^{\prime}$ and $\sigma$ around the transmitter's position are set equal to twice the maximum values of $\epsilon_{r}^{\prime}$ and $\sigma$.

Another important parameter that affects wave propagation in indoor environments is wall thickness. In outdoor ray 
TABLE I: MATERIAL PARAMETERS.

\begin{tabular}{|c||c|c|c|}
\hline MATERIAL & $\epsilon_{r}^{\prime}$ & $\mathrm{c}$ & $\mathrm{d}$ \\
\hline Concrete & 5.31 & 0.0326 & 0.8095 \\
\hline Plasterboard & 2.94 & 0.0116 & 0.7076 \\
\hline Wood & 1.99 & 0.0047 & 1.0718 \\
\hline Glass & 6.27 & 0.0043 & 1.1925 \\
\hline Brick & 3.75 & 0.038 & 0 \\
\hline Air & 1 & 0 & 0 \\
\hline
\end{tabular}

tracing simulations, building walls are modeled as semiinfinite spaces due to the high losses, thus the penetration into buildings or the multiple reflections within the building facets can be omitted. In indoor environments this assumption does not hold, since the walls are thinner than the building facets, and for materials such as plasterboard or wood, the losses are considerably smaller. Hence, objects such as walls, doors and windows are modeled as slabs, and consequently the reflection and the transmission coefficients depend on slabs' thickness. To account for that, we use a third channel which depicts the physical distance between the transmitter and every point in the simulated grid. An example of the distance channel is shown in Fig. 3c. Thus, when a convolutional kernel is applied to a sub-area of the input tensor, apart from detecting the wall type through the first two channels, it can also consider the wall length via the difference between the values of the third channel.

Furthermore, the third channel can also provide a good estimate about the deterioration of the signal over distance. To further enhance the awareness of our model regarding the impact of distance on wave propagation, we add a fourth channel which includes the free space path-loss (FSPL) for each point in the simulated grid. We note that if our model was trained only over one frequency, the fourth channel would have been a simple transformation of the third channel, and thus this information would have been redundant. However, EM DeepRay is trained over multiple frequencies, hence the fourth channel helps our data-driven model to unveil correlations over the frequency and the space domain.

The target output of EM DeepRay is a tensor including the PL at every point of the simulated grid. We note that a similar treatment can be applied for another QoI such as the RSS or the phase delay. As it was shown in [26], each channel of the output tensor corresponds to different sampling height. In this work we use a single ouput channel, representing the PL at the horizontal plane at a height equal to $1.5 \mathrm{~m}$. The target PL values are computed using a ray tracing simulator [31]. The goal is to train a convolutional encoder-decoder which encodes the input tensor and then decodes it as a PL heatmap, i.e., to find a mapping between the material properties, physical distance and frequency space and the PL space.

\section{B. Workflow}

The block diagram of the EM DeepRay, shown in Fig. 4a, preserves the same idea as the initial DeepRay framework [26]. In the initial framework, the simulated results from a ray tracing simulator are leveraged to train a convolutional encoder-decoder to learn how to transform a blueprint of the indoor geometry to a PL heatmap. For each ray tracing simulation we obtain an image, $X_{i}$, representing the geometry of an indoor environment and a tensor, $y_{o}$, comprising the simulated PL values at each point of the indoor geometry. The target PL tensor is converted to a grayscale image, $y_{o, g s}$, and consequently it is resized to a standard size image, $y$. Similarly, the input geometry image it is also resized to a standard size image $X_{c}$. Although a convolutional encoder-decoder can be trained with varying sized images, we found that using standard size images yielded more accurate estimates of the PL. The resized input geometry and PL images are provided as an input-target pair to a convolutional encoder-decoder that performs a pixel-to-pixel prediction, translating the blueprint of an input geometry to a PL heatmap.

The main difference between the two frameworks is the form of the input tensor. The initial DeepRay framework uses a blueprint of the indoor geometry, and the convolutional encoder-decoder learns to identify how different construction materials affect wave propagation, by representing each material with a distinct colour. In this work, we use the blueprint of the input geometry to extract physics-based information that will augment the performance of the convolutional encoderdecoder and allow it to make more accurate predictions. Specifically, as discussed in Section IV-A, for each indoor geometry image, $X_{i}$, we derive an input tensor, $X$, with four channels representing: (i) $\epsilon_{r}^{\prime}$ and (ii) $\sigma$ at each point of the grid, (iii) the physical distance between the transmitter and each point, and (iv) the FSPL for each point. Hence, first we resize the initial input geometry image to a standard size image, and then we use the resized image to compute the values of the four input channels of Fig 3. The fist two channels are derived by corresponding the colours of the blueprint, i.e., different RGB

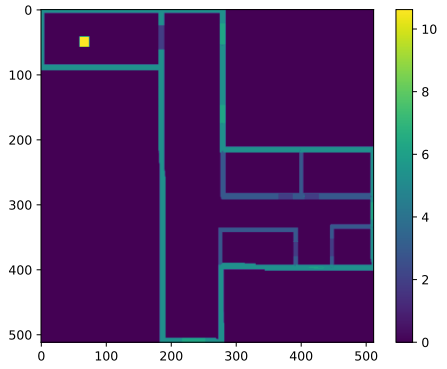

(a) Permittivity channel.

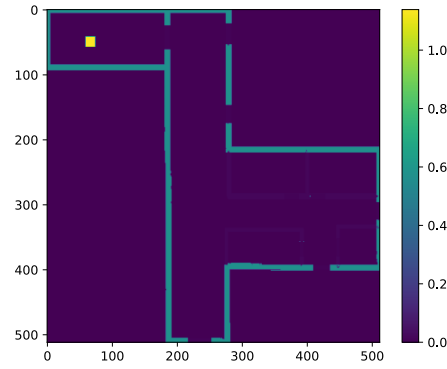

(b) Conductivity channel.

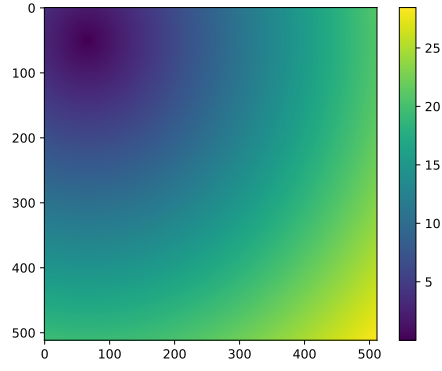

(c) Distance channel.

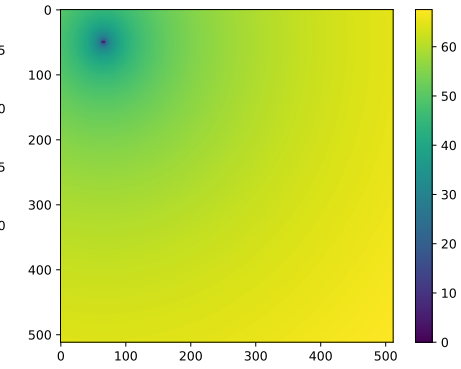

(d) Free space path loss channel.

Fig. 3: Example of the input tensor channels for a random sample at an operating frequency of $2 \mathrm{GHz}$. 


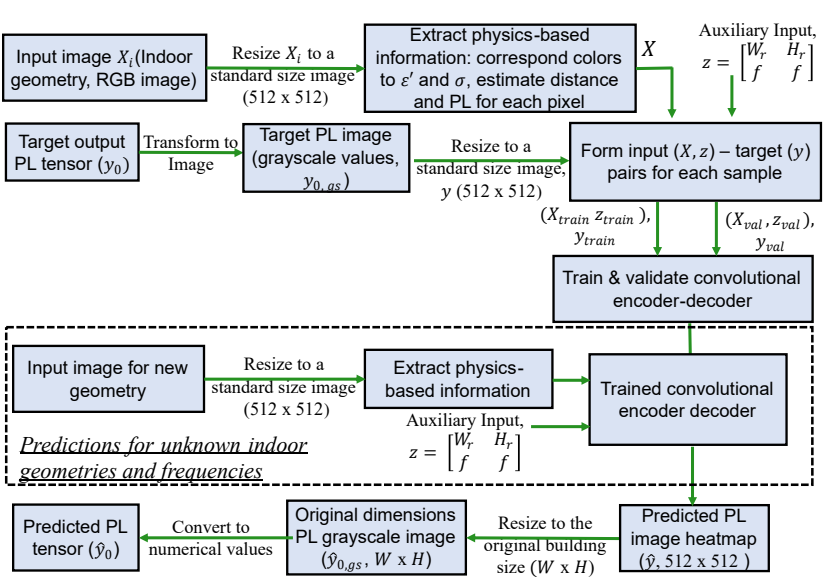

(a) Block diagram of EM DeepRay architecture.

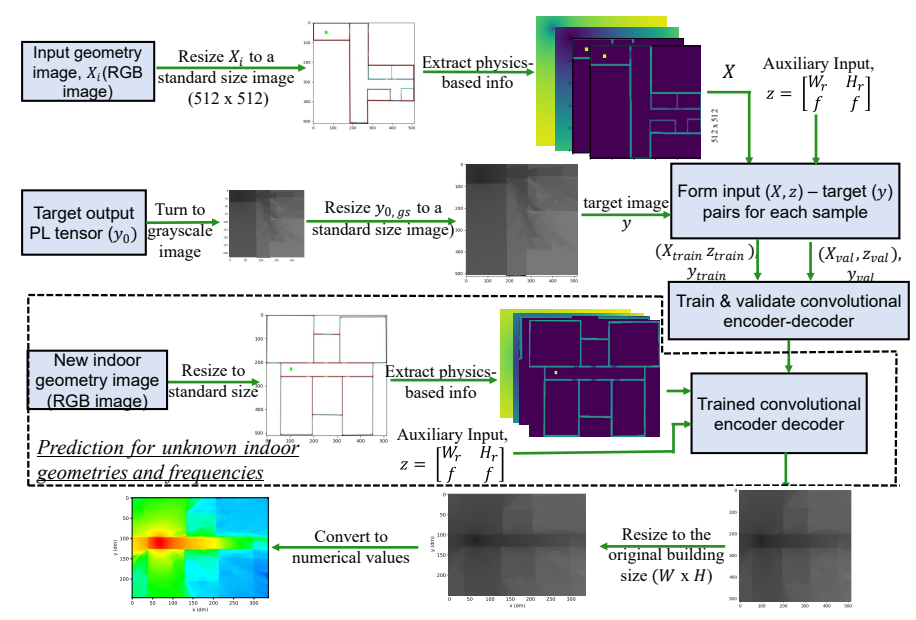

(b) Schematic representation of EM DeepRay functionality.

Fig. 4: EM DeepRay architecture.

pixel values, to the values of Table I. The physical distance, $d_{i, j}$, for the $(i, j)$-th pixel is computed as:

$$
d_{i, j}=R \sqrt{W_{r}\left(x_{T x}-i\right)^{2}+H_{r}\left(y_{T x}-j\right)^{2}}
$$

where $\mathrm{R}$ is the spatial resolution of the ray tracing simulation, $\left(x_{T x}, y_{T x}\right)$ corresponds to the transmitter position in the simulated grid, and $W_{r}, H_{r}=W, H / 512$ are the width and height resizing ratios, respectively. Consequently, the values of the fourth channel are estimated as:

$$
F S P L_{i, j}=20 \log _{10}\left(\frac{4 \pi d_{i, j} f}{c}\right)
$$

A schematic representation of this process is shown in Fig. 4b. As can be observed, the initial input image is resized to a $512 \times 512$ image, and it is used to extract the fourchannel tensor $X$, which is input to the convolutional encoderdecoder. Apart from the input tensor $X$, the convolutional encoder-decoder receives an auxiliary input $z$, which conveys information about the frequency and the resizing ratios, $W_{r}$ and $H_{r}$, of each sample. The details of the incorporation of the auxiliary input with the input tensor are outlined in Section IV-C. The target PL tensor is turned to a grayscale image, assuming values between 0 and 255 , and the grayscale image is resized to have the same size as $X$ (i.e., $512 \times 512$ ). The resized geometry image along with the auxiliary input tensor form a pair of input values, $(X, z)$, while $y$ is the target value. Each input-output pair accounts for a sample used to train the convolutional encoder-decoder.

Once trained, the convolutional encoder-decoder can be used to directly predict the PL for new geometries or frequency bands that are not included in the training data set (dashed box in Figs. 4a and 4b). Predictions for new geometries require only an image of the indoor environment, which is used to extract the physics-based input tensor, as shown in Fig. 4b. The request of an image depicting the input geometry is not undue, since most of existing commercial ray tracing software use such a representation [17], [31]. In addition, our approach can be further facilitated by recent research which allows computer-aided design (CAD) floor plans to be turned into blueprints [32]. We also note that the extraction of the physicsbased input tensor does not entail any complex operations, only if statements and matrix multiplications whose computation requires a few milliseconds.

Given the physics-based input tensor, the convolutional encoder-decoder predicts a PL heatmap image, $\hat{y}$, for the desired input geometry. The predictions of the convolutional encoder-decoder are pixel-wise and the size of the output tensor is $512 \times 512$. Thus, after a prediction, we use a bilinear interpolation to resize the output image back to the initial dimensions $W$ and $H$ of each geometry. As final step, the grayscale PL images are converted to numerical values. The latter simply implies perceiving the elements of the resized input image as $\mathrm{dBm}$ instead of pixel intensity values.

\section{Convolutional encoder-decoder}

Conventionally, CNNs are terminated by consecutive fully connected layers, as shown in Fig. 2. There are three main drawbacks associated with this approach: (i) fully connected layers require a fixed-size input, (ii) they neglect the spatial and the cross-channel correlations and (iii) they are computationally expensive as they have more parameters [33]. That motivated the development of fully convolutional networks $(\mathrm{FCN})$, that are able to perform pixel-to-pixel predictions [33]. In FCN architectures the fully connected layers at the end of the network are replaced by a series of upsampling and convolutional layers. The absence of fully connected layers translates to a smaller number of trainable parameters and a higher efficiency.

A convolutional encoder-decoder is an FCN that consists of two basic components: (i) an encoder, used to downsample the input tensor and compress its context, and (ii) a decoder which is used to recapture the input tensor details and reconstruct it in the form of the target output tensor. Convolutional encoder-decoders may also include skip connections between the encoding and the decoding path, as shown in Fig. 5 (dark yellow arrows), in order to retain information that might be lost during the encoding procedure. In this work we use 


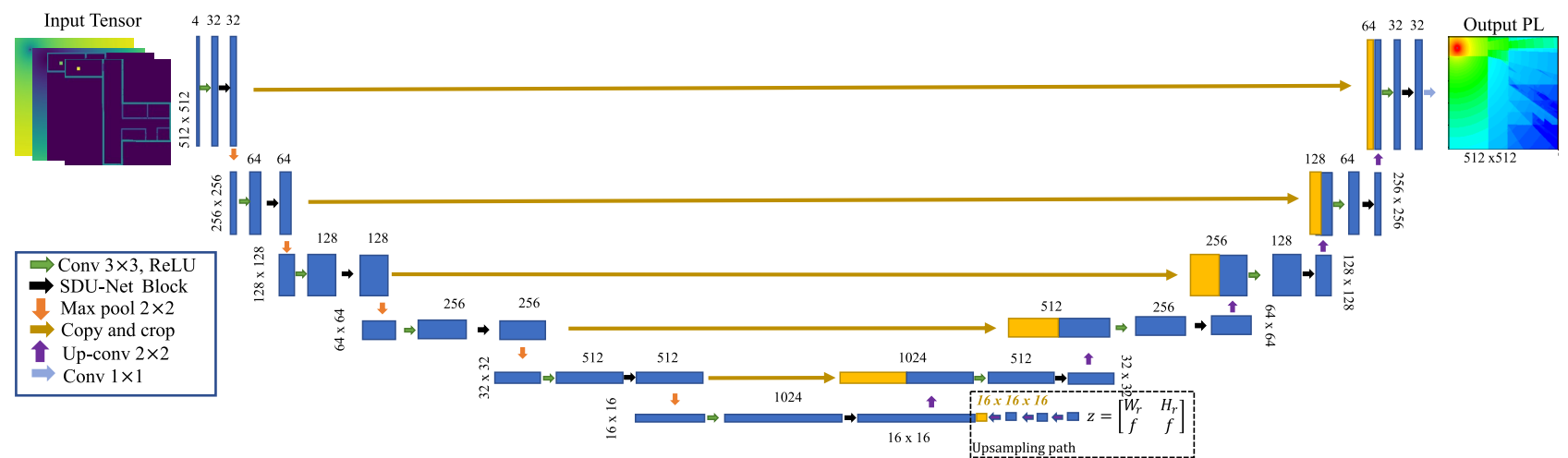

Fig. 5: Convolutional encoder-decoder used in EM DeepRay to encode a physics-based input tensor, and decode it as a PL heatmap.

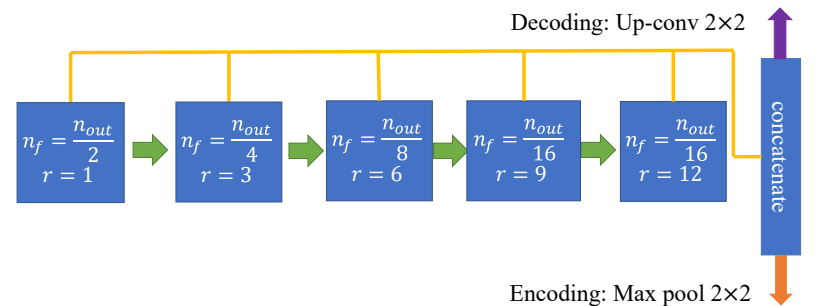

Fig. 6: Structure of the SDU-Net block used at each encoding/decoding layer.

the convolutional encoder-decoder depicted in Fig. 5, which is a slightly modified version of the SDU-Net architecture introduced in [28] (we include an additional upsampling path).

The SDU-Net employed has five encoding and decoding layers, i.e., five downsampling and upsampling operations are applied, denoted by orange and purple arrows at the encoding and the decoding branch, respectively. As can be seen in Fig. 5, the left path of the SDU-Net is used to encode and downsample the input tensor containing information about the indoor geometry, while the right path upsamples the encoded tensor and transforms it to a PL heatmap. At the end of the encoding path, we add an upsampling path used to upsample the auxiliary input $z$. Consecutive upsampling operations are applied to $z$ to increase its size and concatenate it with the encoded representation of the input tensor. That will allow the SDU-Net to understand the operating frequency, and also be aware of the resizing ratio for each sample. This information is not included in the input tensor for computational efficiency, i.e., there is no need to reserve an entire channel for scalar variables. Instead, it is considered during the decoding by fusing the upsampled $z$ with the encoded input tensor just before the decoding procedure begins.

At the encoding path, each layer includes a $3 \times 3$ standard convolution, followed by the SDU-Net block, shown in Fig. 6, and a $2 \times 2$ max pooling with stride equal to 2 . At the decoding branch, the feature map of the previous layer is first upsampled, and then it is concatenated with the feature map of the respective layer of the encoding branch (yellow boxes shown at each layer of the decoding branch). Consequently, a $3 \times 3$ standard convolution is applied to the concatenated tensor, and the resulting feature map is forwarded to the SDUNet block, and moved upwards to the next layer. The number of the convolutional filters is increased or decreased by a factor of two at each encoding or decoding layer, respectively. At the top layer of the decoding branch, a $1 \times 1$ convolution is used after the SDU-Net block to derive the PL heatmap image. The number of the filters for the final $1 \times 1$ convolution is equal to the number of the desired output channels, i.e., one.

The SDU-Net block, shown in Fig. 6, comprises five cascaded atrous convolutions, each one using a different number of filters and a different atrous rate. The feature maps of the different atrous convolutions are concatenated and forwarded to the next layer after being down- or upsampled. Atrous convolution, also known as dilated convolution, is a generalized case of the standard convolution operation. Its popularity has recently increased, since it can be used to efficiently augment the receptive field of each neuron without increasing the number of model parameters [34]. In atrous convolution, the kernel is widened and its sparsity is increased by introducing blank spaces between the kernel elements. The distance between neighboring non-blank elements of the kernel is $r-1$, where $r$ is the atrous rate. The parameters for each atrous convolution are shown in Fig. 6, where $r$ is the atrous rate, and $n_{\text {out }}$ denotes the number of filters at the next layer (which is twice or half the number of filters at the current encoding or decoding layer, respectively).

An example of $3 \times 3$ atrous convolutions with different values of $r$ applied to the image of an indoor environment

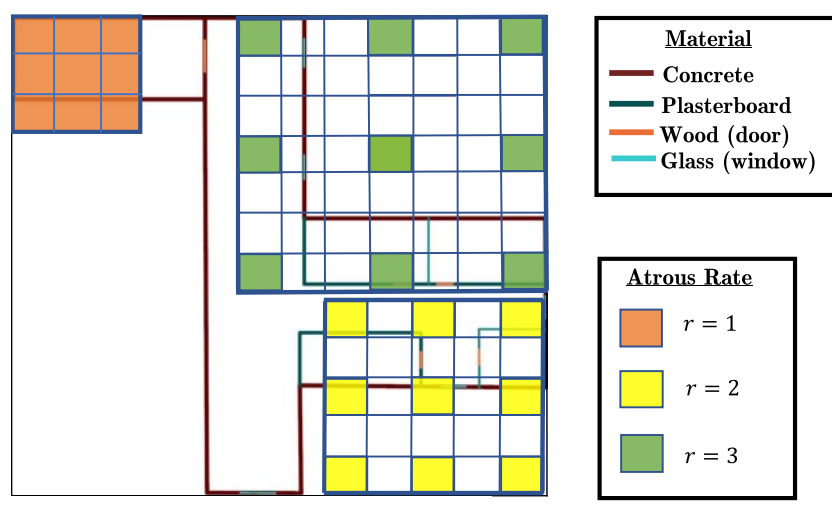

Fig. 7: Example of convolutional filters with different atrous rate $r$ applied to the image of an indoor environment. 
is shown in Fig. 7. It can be observed that as $r$ increases, the receptive field is augmented, since the convolutional kernel has an enhanced field of view over the indoor geometry. Indeed, the area covered by the convolutional kernel with $r=1$ (standard, non-dilated, convolution, orange box) is substantially smaller than that of the kernel with $r=3$. That comes at no increase in the overall computational cost, since the number of trainable parameters remains the same. Furthermore, the use of various atrous convolutions with different atrous rates enables the aggregation of information originating from different spatial scales and the identification of correlations between distant points. Moreover, due to the multiple layers of the SDU-Net architecture, it is also possible to capture multi-scale correlations at different resolutions (since the size of the input tensor is decreased by a factor of two at each encoding layer). The augmented receptive field, and the potential of capturing multi-scale correlations (using various $r$ values) at multiple resolutions (due to the multiple encoding and decoding layers), render the SDUNet an exemplary convolutional encoder-decoder for radio propagation modelling tasks.

\section{Evaluation metrics}

To evaluate the performance of DeepRay we use two error metrics: the mean absolute error (MAE) and the root mean square error (RMSE), defined as follows:

$$
\begin{aligned}
M A E & =\frac{1}{N W H} \sum_{n=1}^{N} \sum_{i=1}^{W} \sum_{j=1}^{H}\left|y_{o,(n)}(\mathrm{i}, \mathrm{j})-\hat{y}_{o,(n)}(\mathrm{i}, \mathrm{j})\right| \\
R M S E & =\sqrt{\frac{\sum_{n=1}^{N} \sum_{i=1}^{W} \sum_{j=1}^{H}\left(y_{o,(n)}(\mathrm{i}, \mathrm{j})-\hat{y}_{o,(n)}(\mathrm{i}, \mathrm{j})\right)^{2}}{N W H}}
\end{aligned}
$$

where $y_{o,(n)}(\mathrm{i}, \mathrm{j})$ and $\hat{y}_{o,(n)}(\mathrm{i}, \mathrm{j})$ are the ray tracing and the predicted PL values for the $n$-th sample at the spatial point $(i, j)$, respectively, and $N$ represents the number of samples. We note that the error is measured at the end of the proposed framework, i.e., it is not pixel-wise but it estimated with respect to the actual PL values.

\section{COMPARISON WITH SIMULATED DATA}

In this section, we proceed to the implementation of EM DeepRay, comparing its path loss predictions to simulated data. To create the synthetic data to train EM DeepRay we use the Ranplan Professional ray tracing simulator, developed by Ranplan Wireless [31]. Ranplan Professional is a robust radio propagation engine, supporting radio propagation simulations for both indoor and outdoor environments. It enables the easy and efficient wireless network design of entire floors or even buildings and it supports a large number of different communication technologies (WiFi, 5G New Radio, IoT). The software has been widely used for actual network planning, which provides us an assurance regarding its reliability.

To train and validate EM DeepRay we conduct ray tracing simulations at 20 different indoor environments. The buildings considered include simple and more complicated indoor environments, with more than 20 sub-rooms in the same building floor, and the walls are made of various construction materials (concrete, brick, plasterboard, wood, and glass) and have various thicknesses. For each building we consider transmitting devices operating at 3 different frequencies: (i) 0.433 , (ii) 2 and (iii) $3.7 \mathrm{GHz}$, which correspond to frequency bands utilized in IoT and $5 \mathrm{G}$ systems. For all the simulations the transmitter antenna is $1.5 \mathrm{~m}$ above the floor, and has an omnidirectional beam pattern. The resolution of the ray tracing simulations is set to 0.1 , i.e., we sample the PL value once per $0.1 \mathrm{~m}$. For each frequency band we run approximately 35 different ray tracing simulations at each building, i.e., $35 \times 3$ samples per building, assuming a different transmitting device position within the indoor geometry for each sample. We use $80 \%$ of the samples to train DeepRay and $20 \%$ to validate its performance. Once the data are split into two sets, the training data set is augmented by flipping the indoor geometry and PL heatmap images left, right and downwards. That allows to effectively increase the training set size by a factor of 3 without conducting any extra simulations. We note that EM DeepRay is validated with samples from known geometries and frequency bands, i.e., from indoor environments and frequency bands included in the training data set, but for unknown transmitting device positions within these environments.

To test EM DeepRay we consider three cases, aiming to explore how well our model: (1) can generalize to new geometries, not included in the training data set, (2) can infer the PL for frequency bands other than those of the training set, and (3) behaves in a combination of (1) and (2). We refer to these cases as Test 1,2, and 3, respectively. The case studies are summarized in Table II, where with the term "known" and "unknown" we indicate either included or not included in the training data set. To implement Test 1, we consider five new indoor environments, generating about 35 samples at each building, for each frequency band of the training data set $(0.433,2$, and $3.7 \mathrm{GHz})$. We underline that EM DeepRay has no prior information regarding the PL distribution within these indoor environments, and it will infer the PL heatmaps based on the weights estimated during the training phase. For Test 2, we assume transmitting devices operating at $866 \mathrm{MHz}$, and we randomly place approximately 35 devices at each one of the 20 buildings used to train and the validate our model. Finally, for Test 3, we consider transmitting devices operating at 866 $\mathrm{MHz}$ at the five buildings used in Test 1, i.e., for buildings and a frequency band not included in the training data set.

Our data-driven model is trained on a Nvidia Quadro RTX 8000 GPU over Tensorflow, using the Adam optimization algorithm for 250 epochs, with the learning rate set to 0.0005 , and a batch size of 4 . The loss function to be optimized is the RMSE. We also explored the use of the MAE, but the performance of our model was slightly worse, thus we present results only using the RMSE as a loss function. During training, the loss function is minimized with respect to $y$ and $\hat{y}$, i.e., based on the pixel intensity values of the ray tracing and the predicted PL images. However, the error metrics are estimated with respect to the ray tracing and the predicted actual PL values (i.e., between $y_{o}$ and $\hat{y}_{o}$, and they are in 


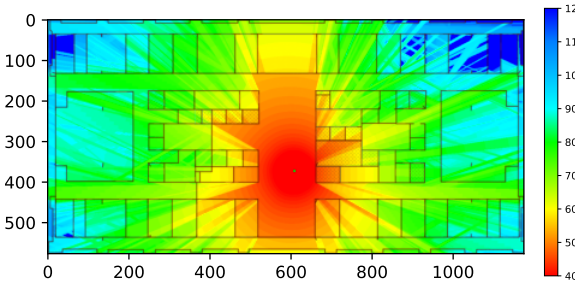

(a) ray tracing.

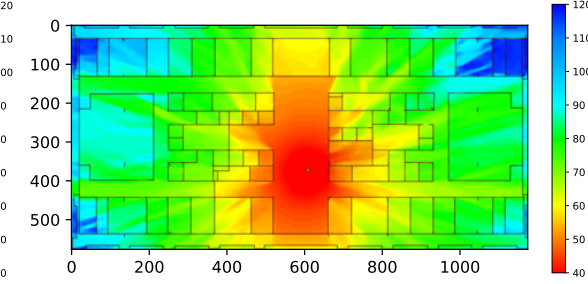

(b) EM DeepRay.

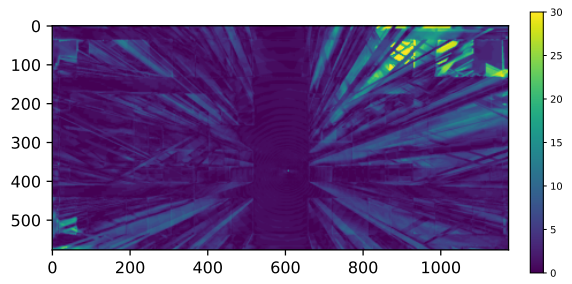

(c) Error Map.

Fig. 8: Comparison between the PL heatmap simulated through (a) ray tracing and predicted by (b) EM DeepRay for a validation set sample at $433 \mathrm{MHz}$ (known building and frequency, but unknown transmitter position). The (c) error map depicts the absolute error between (a) and (b).

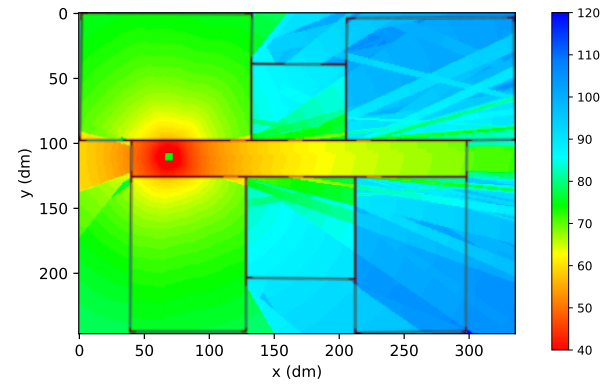

(a) ray tracing.

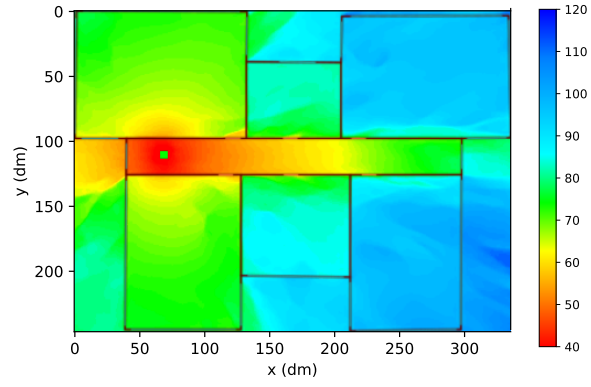

(b) EM DeepRay.

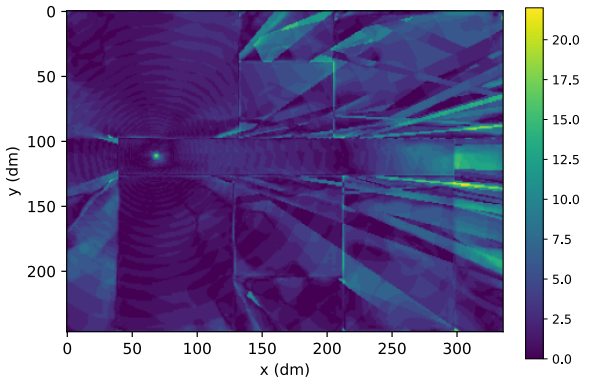

(c) Error Map.

Fig. 9: Comparison between the PL heatmap simulated through (a) ray tracing and predicted by (b) EM DeepRay for a Test set 1 sample at $2 \mathrm{GHz}$ (known frequency band, but unknown indoor geometry). The error map (c) depicts the absolute error between (a) and (b).

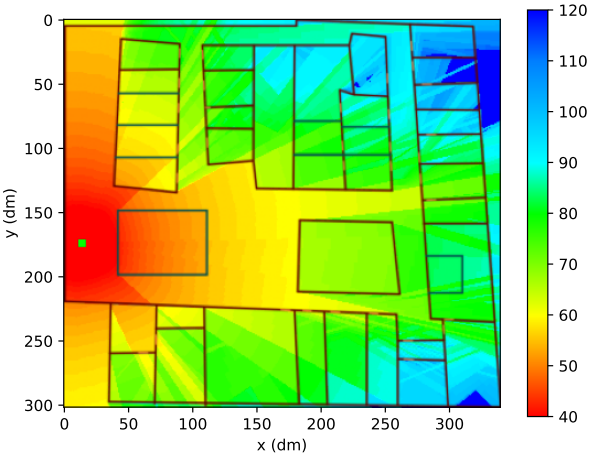

(a) ray tracing.

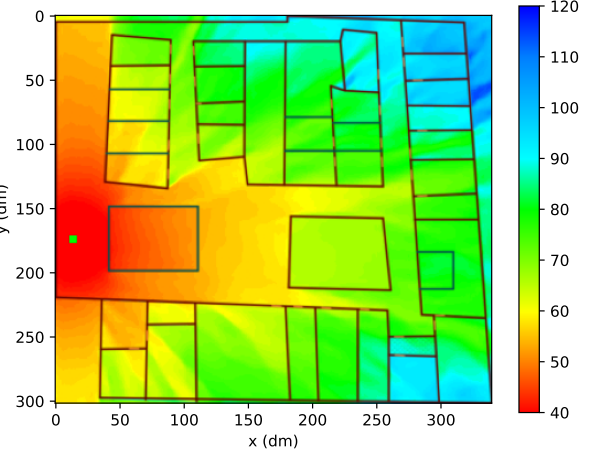

(b) EM DeepRay.

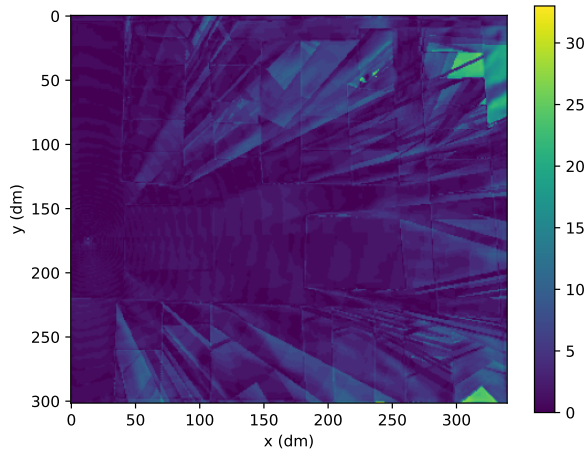

(c) Error Map.

Fig. 10: Comparison between the PL heatmap simulated through (a) ray tracing and predicted by (b) EM DeepRay for a Test set 2 sample at $868 \mathrm{MHz}$ (known indoor geometry, but unknown frequency band). The error map (c) depicts the absolute error between (a) and (b).

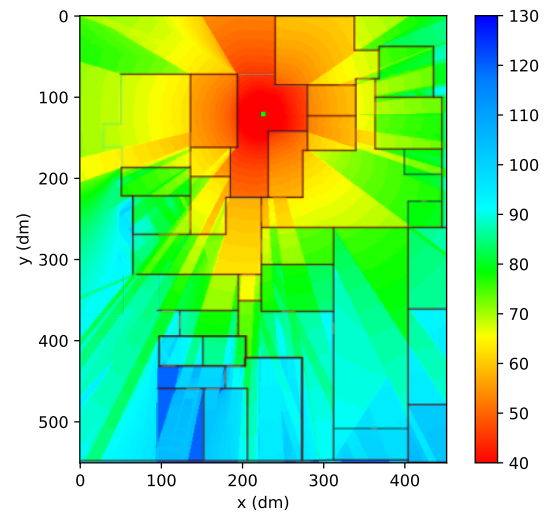

(a) ray tracing.

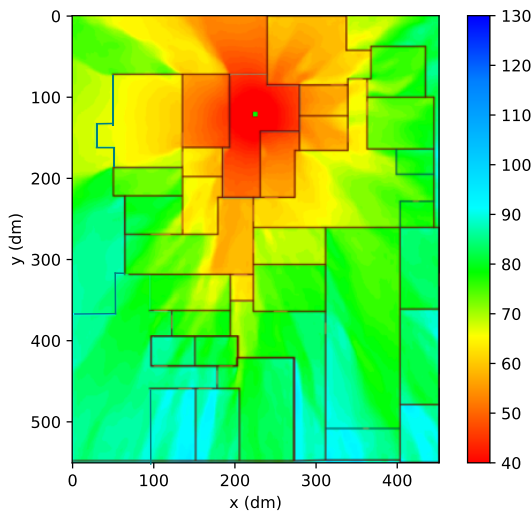

(b) EM DeepRay.

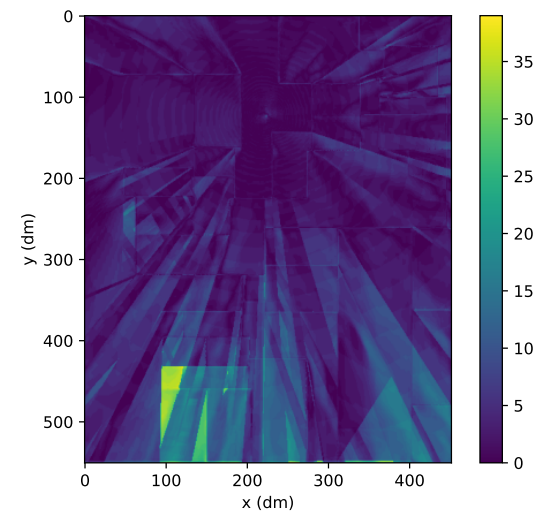

(c) Error Map.

Fig. 11: Comparison between the PL heatmap simulated through (a) ray tracing and predicted by (b) EM DeepRay for a Test set 3 sample at $868 \mathrm{MHz}$ (unknown indoor geometry and frequency band). The error map (c) depicts the absolute error between (a) and (b). 
TABLE II: CASE STUDIES: KNOWN AND UNKNOWN INDICATES INCLUDED OR NOT INCLUDED IN THE TRAINING DATA SET.

\begin{tabular}{|c||c|c|c|}
\hline & Transmitter Position & Geometry & Frequency \\
\hline \hline Training & Known & Known & Known \\
\hline Validation & Unknown & Known & Known \\
\hline Test 1 & Unknown & Unknown & Known \\
\hline Test 2 & Unknown & Known & Unknown \\
\hline Test 3 & Unknown & Unknown & Unknown \\
\hline
\end{tabular}

$\mathrm{dBm})$. The training takes almost 2 hours, while a prediction for a single sample requires about 100 milliseconds, including the extraction of the physics-based input tensor.

The overall error metrics between the simulated and the predicted PL values for all the case studies are presented in Table III. The error metrics are negligible for the training set, with the RMSE and the MAE being equal to 1.2 and 0.99 $\mathrm{dBm}$, respectively. That indicates that the proposed framework can indeed learn to translate a map of physics-based information to a PL heatmap. The validation set error metrics are approximately $3 \mathrm{dBm}$ larger than these of the training set, but they still assume low values. More importantly, the good resemblance between the simulated and the predicted data is also preserved for the three different test cases. In particular, Test 1 and 2 yield approximately an RSME of $5 \mathrm{dBm}$ and an MAE of $3.8 \mathrm{dBm}$, while for Test 3 the respective values are 5.6 and $4.5 \mathrm{dBm}$. Thus, in addition to learning to infer the signal attenuation within a given geometry and frequency band, our model is generalizable, and it can provide accurate estimates of the PL for new indoor geometries and frequencies.

In Figs. 8, 9, 10, and 11, we visualize the results for a random sample from the validation, the Test 1,2 , and 3 set, respectively. Figures 8a-11a show the simulated ray tracing "ground-truth" for each sample, while the corresponding PL heatmap predicted by EM DeepRay is illustrated in Figs. 8b11b. We note that DeepRay outputs solely the PL heatmap, and in order to illustrate the input geometry we consequently impose its blueprint on top of the PL heatmap. As can be observed, the similarity between the simulated and the predicted PL heatmaps is very high for all the cases. The absolute error maps in Figs. 8c-11c depict the absolute error, $\left|y_{o}(\mathrm{i}, \mathrm{j})-\hat{y}_{o}(\mathrm{i}, \mathrm{j})\right|$, at each spatial point $(i, j)$ within the simulated indoor environment. A common trend is that the predicted PL exhibits the largest absolute errors at positions far away from the transmitting device. For instance, as can be seen in Fig. 11c, the largest errors are found on the lower left area of the floor. That is more than 40 and 10 meters away from the transmitter, in the horizontal and vertical directions, respectively. This is encouraging, since when a user is located far away from a transmitter it is likely that they are served by another device, and thus these areas do not have significant impact on network design decisions. This observation also indicates that MAE is a more appropriate error metric, since RMSE penalizes large errors, which in our case occur in areas of low interest.
TABLE III: EM DEEPRAY PERFORMANCE FOR EACH CASE STUDY.

\begin{tabular}{|c||c|c|c|}
\hline & RMSE $(\mathrm{dBm})$ & MAE $(\mathrm{dBm})$ & Samples \\
\hline \hline Training & 1.2 & 0.99 & 1680 \\
\hline Validation & 4.21 & 3.26 & 420 \\
\hline Test 1 & 5.05 & 3.86 & 525 \\
\hline Test 2 & 5.00 & 3.88 & 700 \\
\hline Test 3 & 5.65 & 4.53 & 165 \\
\hline
\end{tabular}

\section{COMPARISON WITH MEASURED DATA AND} CALIBRATION THROUGH TRANSFER LEARNING

In the previous section, we demonstrated that EM DeepRay can be trained with physics-based data of various indoor environments and eventually learn to predict the PL within them. Once trained, EM DeepRay can be used as standalone propagation model to furnish estimates of the PL for an arbitrarily complex indoor geometry at a given frequency. The credibility of our model highly depends on the data used to develop it. These data are only an approximation of the actual signal attenuation and they are themselves subject to errors. However, the purpose of an ML-based propagation model is to deliver results that closely resemble actual rather than synthetic data.

In this subsection, we address this issue by outlining a simple, yet efficient, approach that allows the calibration of EM DeepRay through transfer learning. The aim of transfer learning is to leverage knowledge accrued to tackle a certain problem, and employ it to solve a different problem, which is related back to the initial problem [24]. In our case, the first problem is the design of an ML-based propagation model that can predict the PL in indoor environments, whilst the second problem is to make the ML-based propagation model realistic. The motivation behind the use of transfer learning, instead of training EM DeepRay simultaneously with synthetic and measured data, is that measured data are scarce and sparse. Indeed, measurement campaigns are time-consuming and expensive, and usually the results from large scale campaigns are not publicly available. In addition, typically only a small number of measured RSS values is available for each indoor environment, since these campaigns are realized by moving the receiving device around within the area of interest and recording the RSS at some sparse points.

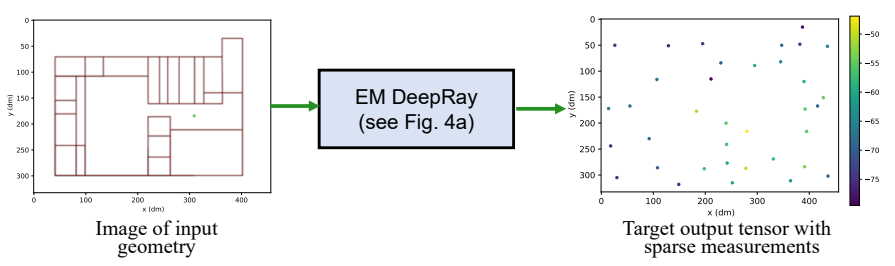

Fig. 12: Calibration framework; the dots in the target tensor correspond to points in the indoor geometry at which the RSS is measured, and their color represents the RSS intensity.

Hence, if a data set with both simulated and measured data is used to train an ML propagation model, it would comprise a limited number of measurement scenarios (compared to the simulated ones) with only a few recordings per scenario. That would render the training challenging, as it would be necessary 
to find a balance between the importance of the measured over the simulated data. Instead, treating the calibration as a distinct problem enables handling a limited quantity of sparse data in a more efficient manner. In particular, to calibrate our model we use the same framework shown in Fig. 4a, however, the target output tensor is now different. As shown in Fig. 12, for the calibration the target output tensor comprises the recorded measurements at some points within the simulated grid, instead of simulated ray tracing PL values. To distinguish the target output tensors of the calibration framework we use the subscript $c$, referring to the initial and the resized target output as $y_{c, 0}$ and $y_{c}$, respectively. Thus, the calibration process constitutes at retraining the pre-trained model obtained in Section $\mathrm{V}$ to adapt its PL predictions and match the measured PL values of $y_{c}$.

A significant difference between the target output tensors of the frameworks, shown in Figs. 4a and 12, is that most elements of $y_{c, 0}$ and $y_{c}$ are zeros, i.e. no measurements are recorded for these points. For that reason, it is not possible to use the same loss function as the one used in Section V. More specifically, the operation of EM DeepRay is equivalent to applying a mapping function $f$, parametrized through a set of learnable weighs $\Theta$, to the initial input tensor $X$ in order to derive the output PL image heatmap, i.e., $\hat{y}=f(X \mid \Theta)$. The values of $\Theta$ are computed iteratively, such as to minimize the difference between the pixel intensity of the ray tracing "ground truth" and the predicted PL image heatmap:

$$
\min _{\Theta}\left(\frac{1}{N W H} \sum_{n, i, j=1}^{N, W, H}\left(y_{(n)}(\mathrm{i}, \mathrm{j})-\hat{y}_{(n)}(\mathrm{i}, \mathrm{j})\right)^{2}\right)^{1 / 2}
$$

where $y_{(n)}(\mathrm{i}, \mathrm{j})$ and $\hat{y}_{(n)}(\mathrm{i}, \mathrm{j})$, are the ray tracing and the predicted pixel intensity values, respectively, for the $n$-th sample at pixel $(i, j)$. Evidently, if $y$ is zero at most points, for (7) to be minimized, the values of $\Theta$ should be selected such as $\hat{y}$ is also zero at these points. Thus, using the same loss function for $y_{c}$, which has a few non-zero points, will force the pre-trained EM DeepRay to breakdown. To overcome this limitation, during the calibration when (7) is computed for $y_{c}$ and $\hat{y}_{c}$, their difference is multiplied with a term $Q_{c}$ defined as:

$$
Q_{c}(i, j)= \begin{cases}1 & \text { if } y_{c}(\mathrm{i}, \mathrm{j}) \neq 0 \\ 0 & \text { if } y_{c}(\mathrm{i}, \mathrm{j})=0\end{cases}
$$

and hence the only elements that contribute to the loss function are those for which measured data exist. That allows us to slightly modify the pre-estimated weights $\Theta$ during the calibration (retraining) of our model, and compute some new weights $\Theta^{\prime}$, in a way that $\hat{y}_{c}=f\left(X \mid \Theta^{\prime}\right)$ matches only the nonzero values of $y_{c}$. We note that the role of $Q_{c}$ is to consider only the non-zero points of $y_{c}$ during the computation of $\Theta^{\prime}$, however, that does not imply that the predictions for other points of the simulation grid will remain intact. That is to say, that $\Theta^{\prime}$ is estimated based only on a few points, but the change of the weights affects the predictions for the entire grid.

To demonstrate the effectiveness of our approach, we generate the indoor geometry of [35], and provide it as an input to

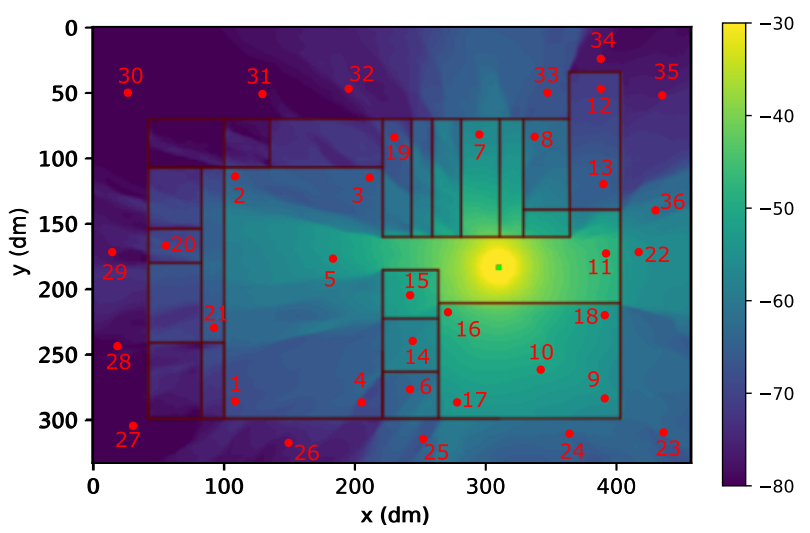

(a) DeepRay RSS prediction before calibration for the indoor geometry of [35].

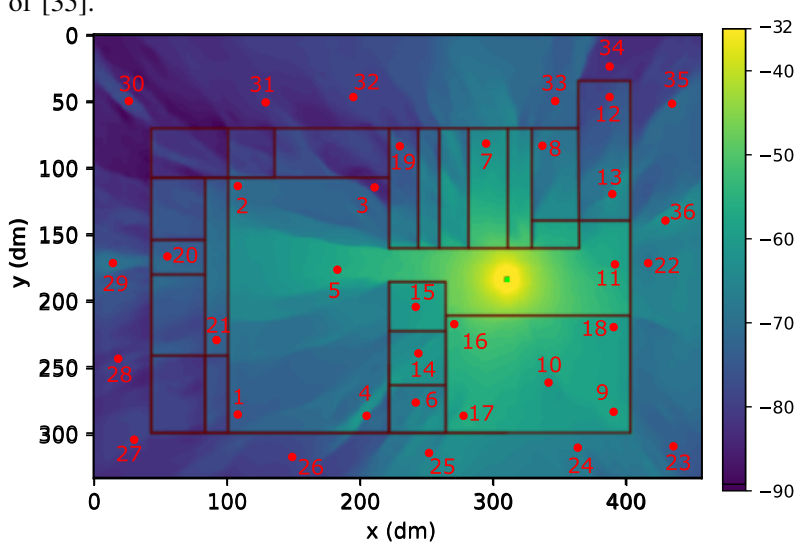

(b) DeepRay RSS prediction after calibration for the indoor geometry of [35].

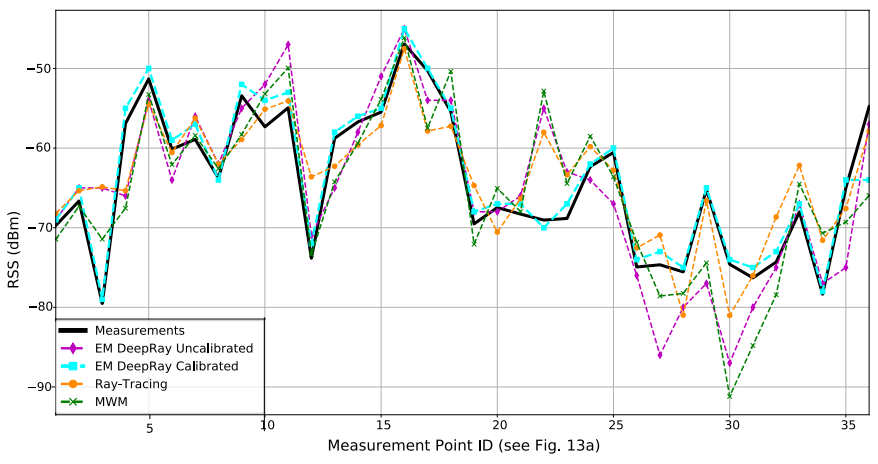

(c) Comparison between simulated and measured RSS

Fig. 13: Predicted RSS, and comparison with measured data [35].

DeepRay. The operating frequency is $868 \mathrm{MHz}$. In the absence of detailed information regarding the material types, all the walls found within the geometry are assumed to be made of concrete and are $10 \mathrm{~cm}$ thick. Again, the input geometry image is resized and it is used to derive the physics-based input tensor. The target output, $y_{c, o}$ is turned into an image and it is also resized to have the same size as the input tensor. The target output tensor has only 36 non-zero points, i.e., we have measurements for 36 points within the simulated geometry. We note that the measurements usually depict the RSS, while EM DeepRay predicts the PL. To account for that, the elements of $y_{o, c}$ are calculated as $T x_{\text {power }}-R S S_{\text {measured }}$, where $T x_{\text {power }}$ is the transmitting power. Then, the two tensors along with the 
TABLE IV: ERROR OF EACH PROPAGATION MODEL COMPARED TO MEASUREMENTS.

\begin{tabular}{|c||c|c|c|c|}
\hline & EM DeepRay & RT & MWM & $\begin{array}{c}\text { Calibrated } \\
\text { EM DeepRay }\end{array}$ \\
\hline \hline RMSE (dBm) & 6.1 & 5.24 & 6.21 & 1.69 \\
\hline MAE (dBm) & 4.65 & 4.33 & 4.78 & 1.22 \\
\hline $\begin{array}{c}\text { Computational } \\
\text { time (sec) }\end{array}$ & 0.09 & 1280 & 194 & 15,1 \\
\hline
\end{tabular}

auxiliary vector are passed to the SDU-Net which performs a pixel-to-pixel regression, updating its weights considering only the non-zero points of the target output image tensor, $y_{c}$, as discussed earlier. The SDU-Net is retrained using the Adam optimization algorithm. To avoid abrupt changes of $\Theta$, we train the SDU-Net over fewer epochs and we use a smaller learning rate than that of Section V. Expressly, the learning rate is equal to $10^{-5}$ and the training lasts for 50 epochs. Finally, to prevent overfitting we add an $L_{2}$ regularization at each convolutional layer, setting the regularization parameter equal to 0.01 [24]. The calibration procedure takes 15 seconds, since the SDU-Net is retrained over a single sample. Once the SDU-Net is retrained, we obtain the predicted PL tensor $\hat{y}_{c, o}$ and we subtract it from the transmitting power to derive the predicted RSS values.

The predicted signal level by DeepRay is benchmarked against ray tracing and the the multi-wall model (MWM) [35]. The RSS predicted by DeepRay before calibration is shown in Fig. 13a, with the blueprint of the input geometry printed on top of it. The predicted RSS after calibration is shown in Fig 13b. The red dots in Figs. 13a and 13b, numbered from 1 to 36 , correspond to the points at which the measurements were taken. The measured, simulated and predicted RSS for each point is shown in Fig. 13c, where the $x$-axis signifies the corresponding red dot position shown in Fig. 13a. A comparison of the error metrics for the three propagation models is presented in Table IV. Prior to calibration, ray tracing demonstrates the smallest errors yielding an RSME and the MAE being equal to 5.2 and $4.3 \mathrm{dBm}$, respectively. The calculated error values for EM DeepRay and MWM are approximately $1 \mathrm{dBm}$ larger. We remark that EM DeepRay is not trained in this geometry and it can directly make predictions by just using a blueprint. A significant advantage of EM DeepRay over both ray tracing and MWM is the substantially computational time required to compute the PL values. Indeed, our model's estimation is based solely on multiple matrix multiplications that can be executed within a few milliseconds in a GPU. On the other hand, ray tracing requires to determine all the rays that can reach a receiving point, while MWM needs to estimate the number of walls between the transmitter and each point in the simulation grid through Bresenham's line algorithm [36]. The substantial difference in the computational time can be critical when it comes to the optimal network planning, where multiple simulations are conducted within the same geometry, aiming at meeting certain quality of service requirements.

After the calibration, the predicted RSS values show a close correspondence with the measured data, exhibiting an RMSE and an MAE equal to 1.69 and $1.22 \mathrm{dBm}$, respectively. The computational time for the calibrated EM DeepRay, shown in
TABLE V: CALIBRATION RESUlTS USING A SMALLER NUMBER OF RANDOM TRAINING POINTS, AND ESTIMATING THE ERROR WITH RESPECT TO THE REMAINING MEASUREMENT POINTS.

\begin{tabular}{|c||c|c|c|c|}
\hline $\begin{array}{c}\text { Number of } \\
\text { Training Points }\end{array}$ & 4 & 8 & 16 & 27 \\
\hline \hline RMSE $(\mathrm{dBm})$ & 4.46 & 3.78 & 3.32 & 2.57 \\
\hline MAE $(\mathrm{dBm})$ & 3.62 & 3.24 & 2.45 & 2.01 \\
\hline
\end{tabular}

Table IV, even if we include the training time (15 seconds), it remains much lower than that of ray tracing and MWM. More importantly, as pointed out earlier and can be seen in Fig. 13b, due to the new weights $\Theta^{\prime}$ the predicted RSS values are different for the entire grid, and not just for the few measurement points. For instance the RSS was underestimated for the entire outdoor area on the left side of the building, but after the computation of $\Theta^{\prime}$ the signal for the entire area (and not only for points 27, 28, 29 and 30) assumes higher values. To further test our assumption that due to the calibration the predicted RSS values for the entire grid are improved, we calibrate EM DeepRay using only a fraction of the total 36 measurement points, and measuring the error with respect to the remaining points. The results are shown in Table V, where we consider cases in which EM DeepRay is calibrated using randomly only $4,8,16$, and 27 out of the 36 measurements, and the error metrics are estimated with respect to the remaining 32, 28, 20 and, 9 measurement points, respectively. As can be seen, the predictions for the rest of the grid are improved, and the accuracy of our model is increased even using a small number of points during the calibration.

\section{CONCLUSION}

Wireless communication system design requires robust and expedient propagation modeling tools to ensure an optimal network performance. In this paper we introduced a generalizable and realistic ML-based radio propagation modeling framework for indoor environments. Our model exploits physics-based information extracted from the blueprint of an indoor environment to predict the PL within an indoor area of interest. Unlike previous work in the field, the predictions of our model are not restricted to indoor geometries included in the training set, but it can be employed to readily predict the PL for an arbitrarily complex indoor environment. Our results indicate that our model can very well replicate the PL simulated by a ray-tracer, with the distinct advantage of a considerably lower computational time. More importantly, in this work we presented a calibration method that allows our data-driven model to adjust its weights in order to make predictions that are closer to measured rather than synthetic data. We demonstrated that after calibration, which only takes a few seconds, our model can provide estimates of the RSS that resemble actual measured data with outstanding fidelity. Our work tackles two fundamental problems of ML-based propagation modelling (generalizability and credibility), and it paves the way for the establishment of a family of completely automated ML-based propagation models that will assist the deployment of next generation wireless networks. 


\section{REFERENCES}

[1] A. Čolaković and M. Hadžialić, "Internet of Things (IoT): A review of enabling technologies, challenges, and open research issues," Computer Networks, vol. 144, pp. 17-39, 2018.

[2] M. Agiwal, A. Roy, and N. Saxena, "Next generation 5G wireless networks: A comprehensive survey," IEEE Commun. Surveys Tuts., vol. 18, no. 3, pp. 1617-1655, 2016.

[3] T. S. Rappaport, Y. Xing, O. Kanhere, S. Ju, A. Madanayake, S. Mandal, A. Alkhateeb, and G. C. Trichopoulos, "Wireless communications and applications above $100 \mathrm{GHz}$ : Opportunities and challenges for 6G and beyond," IEEE Access, vol. 7, pp. 78 729-78 757, 2019.

[4] A. Zappone, M. Di Renzo, and M. Debbah, "Wireless networks design in the era of deep learning: Model-based, AIbased, or both?" IEEE Trans. Commun., vol. 67, no. 10, pp. 7331-7376, 2019.

[5] B. Ma, W. Guo, and J. Zhang, "A survey of online data-driven proactive 5G network optimisation using machine learning," IEEE Access, vol. 8, pp. 35 606-35 637, 2020.

[6] Y. Sun, M. Peng, Y. Zhou, Y. Huang, and S. Mao, "Application of machine learning in wireless networks: Key techniques and open issues," IEEE Commun. Surveys Tuts., vol. 21, no. 4, pp. 3072-3108, 2019.

[7] J. Wang, C. Jiang, H. Zhang, Y. Ren, K.-C. Chen, and L. Hanzo, "Thirty years of machine learning: The road to paretooptimal wireless networks," IEEE Commun. Surveys Tuts., 2020.

[8] M. Chen, U. Challita, W. Saad, C. Yin, and M. Debbah, "Artificial neural networks-based machine learning for wireless networks: A tutorial," IEEE Commun. Surveys Tuts., vol. 21, no. 4, pp. 3039-3071, 2019.

[9] C. Zhang, P. Patras, and H. Haddadi, "Deep learning in mobile and wireless networking: A survey," IEEE Commun. Surveys Tuts., vol. 21, no. 3, pp. 2224-2287, 2019.

[10] A. Seretis and C. D. Sarris, "An overview of machine learning techniques for radiowave propagation modeling," arXiv preprint arXiv:2101.11760, 2021.

[11] J. Zhang, L. Liu, Y. Fan, L. Zhuang, T. Zhou, and Z. Piao, "Wireless channel propagation scenarios identification: A perspective of machine learning," IEEE Access, vol. 8, pp. $47797-47806,2020$.

[12] E. Damosso, L. M. Correia, et al., "COST action 231: Digital mobile radio towards future generation systems: Final report," European commission, 1999.

[13] R. Janaswamy, Radiowave propagation and smart antennas for wireless communications. Springer Science \& Business Media, 2001.

[14] T. S. Rappaport, Y. Xing, G. R. MacCartney, A. F. Molisch, E. Mellios, and J. Zhang, "Overview of millimeter wave communications for fifth-generation (5G) wireless networks-with a focus on propagation models," IEEE Trans. Antennas Propag., vol. 65, no. 12, pp. 6213-6230, 2017.

[15] V. Abhayawardhana, I. Wassell, D. Crosby, M. Sellars, and M. Brown, "Comparison of empirical propagation path loss models for fixed wireless access systems," in 2005 IEEE 61st Vehicular Technology Conference, IEEE, vol. 1, 2005, pp. 7377.

[16] T. K. Sarkar, Z. Ji, K. Kim, A. Medouri, and M. SalazarPalma, "A survey of various propagation models for mobile communication," IEEE Antennas Propag. Magazine, vol. 45, no. 3, pp. 51-82, 2003.

[17] D. He, B. Ai, K. Guan, L. Wang, Z. Zhong, and T. Kürner, "The design and applications of high-performance ray-tracing simulation platform for $5 \mathrm{G}$ and beyond wireless communications: A tutorial," IEEE Commun. Surveys Tuts., vol. 21, no. 1, pp. 10-27, 2018.

[18] Huawei-Carrier, "Indoor 5G networks [white paper]," 2018.

[19] L. Azpilicueta, M. Rawat, K. Rawat, F. M. Ghannouchi, and F. Falcone, "A ray launching-neural network approach for radio wave propagation analysis in complex indoor environments," IEEE Trans. Antennas Propag., vol. 62, no. 5, pp. 2777-2786, 2014.

[20] X. Zhang, X. Shu, B. Zhang, J. Ren, L. Zhou, and X. Chen, "Cellular network radio propagation modeling with deep convolutional neural networks," in Proceedings of the 26th ACM SIGKDD International Conference on Knowledge Discovery \& Data Mining, 2020, pp. 2378-2386.

[21] O. Perrault, J.-P. Rossi, and T. Balandier, "Predicting field strength with a neural ray-tracing model," in Proceedings of GLOBECOM'96. 1996 IEEE Global Telecommunications Conference, IEEE, vol. 2, 1996, pp. 1167-1171.

[22] I. Popescu, D. Nikitopoulos, P. Constantinou, and I. Nafornita, "ANN prediction models for outdoor environment," in 2006 IEEE 17th International Symposium on Personal, Indoor and Mobile Radio Communications, IEEE, 2006, pp. 1-5.

[23] S. P. Sotiroudis, S. K. Goudos, and K. Siakavara, "Deep learning for radio propagation: Using image-driven regression to estimate path loss in urban areas," ICT Express, vol. 6, no. 3, pp. $160-165,2020$.

[24] I. Goodfellow, Y. Bengio, A. Courville, and Y. Bengio, Deep learning, 2. MIT press Cambridge, 2016, vol. 1.

[25] I. Popescu, D. Nikitopoulos, I. Nafornita, and P. Constantinou, "ANN prediction models for indoor environment," in 2006 IEEE International Conference on Wireless and Mobile Computing, Networking and Communications, IEEE, 2006, pp. 366-371.

[26] S. Bakirtzis, K. Qiu, J. Zhang, and I. Wassell, "DeepRay: Deep learning meets ray-tracing," in 2022 16th European Conference on Antennas and Propagation (EuCAP), 2022.

[27] L. Bai, C.-X. Wang, J. Huang, Q. Xu, Y. Yang, G. Goussetis, J. Sun, and W. Zhang, "Predicting wireless mmWave massive MIMO channel characteristics using machine learning algorithms," Wireless Communications and Mobile Computing, vol. 2018, 2018.

[28] S. Wang, S.-Y. Hu, E. Cheah, X. Wang, J. Wang, L. Chen, M. Baikpour, A. Ozturk, Q. Li, S.-H. Chou, et al., "U-net using stacked dilated convolutions for medical image segmentation," arXiv preprint arXiv:2004.03466, 2020.

[29] S. Bakirtzis, T. Hashimoto, and C. D. Sarris, "FDTD-based diffuse scattering and transmission models for ray tracing of millimeter-wave communication systems," IEEE Trans. Antennas Propag., vol. 69, no. 6, pp. 3389-3398, 2020.

[30] ITU-Rec, "Recommendation P.2040-1, Effects of building materials and structures on radiowave propagation above about 100 MHz," Int. Telecommun. Union, Geneva, Switzerland, 2015.

[31] (2021) Ranplan Wireless, Ranplan Professional. [Online]. Available: https://ranplanwireless.com/.

[32] Z. Zeng, X. Li, Y. K. Yu, and C.-W. Fu, "Deep floor plan recognition using a multi-task network with room-boundaryguided attention," in Proceedings of the IEEE/CVF International Conference on Computer Vision, 2019, pp. 9096-9104.

[33] J. Long, E. Shelhamer, and T. Darrell, "Fully convolutional networks for semantic segmentation," in Proceedings of the IEEE conference on computer vision and pattern recognition, 2015, pp. 3431-3440.

[34] L.-C. Chen, G. Papandreou, F. Schroff, and H. Adam, "Rethinking atrous convolution for semantic image segmentation," arXiv preprint arXiv:1706.05587, 2017.

[35] S. Hosseinzadeh, H. Larijani, K. Curtis, A. Wixted, and A. Amini, "Empirical propagation performance evaluation of LoRa for indoor environment," in 2017 IEEE 15th International Conference on Industrial Informatics (INDIN), IEEE, 2017, pp. 26-31.

[36] S. Hosseinzadeh. (2021). "Multi wall (COST231) signal propagation models + python code," [Online]. Available: https : //www. mathworks . com/matlabcentral/fileexchange/61340 multi-wall-cost231-signal-propagation-models-python-code. 